Journal of Engineering and Applied Sciences 14 (Special Issue 6): 9224-9231, 2019

ISSN: 1816-949X

(C) Medwell Journals, 2019

\title{
Effects of Thermophoresis and Brownian Motion on Boundary Layer Flow over a Linearly Stretching Sheet Saturated by a Nanofluid with the Presence of Magnetic Field and Chemical Reaction
}

\author{
${ }^{1}$ A.G. Madaki, ${ }^{2} \mathrm{M}$. Abdulhameed, ${ }^{1} \mathrm{M}$. Mohamad, ${ }^{1} \mathrm{M}$. Ali and ${ }^{1} \mathrm{R}$. Roslan \\ ${ }^{1}$ Faculty of Science, Technology and Human Development, Universiti Tun Hussein Onn Malaysia, \\ 86400 Batu Pahat, Johor, Parit Raja, Malaysia \\ ${ }^{2}$ School of Science and Technology, Federal Polytechnic, P.M.B. 0231 Bauchi, Nigeria
}

\begin{abstract}
The two-dimensional steady boundary layer flow of a nanofluid past a stretching sheet with a convective boundary condition in the presence of chemical reaction has been studied. The equation of volume fraction concentration consists of the Brownian motion and the thermophoresis effects. The governing equations are simplified via. boundary value approximation and some similarity variables and solved using the Runge-Kutta fourth order method with shooting technique and Homotopy Perturbation Method (HPM) with Pade approximation (HPM-Pade). All the salient parameters on the temperature and concentration profiles have been studied and details are given graphically. Thus, the results found in this study are being compared with the previously published study where an excellent agreement is achieved. As presented on tables. It is found that the reduced Nusselt number is a decreasing function of both generative and destructive chemical reaction - While the reduced Sherwood number is a decreasing function when chemical reaction parameter, $\bullet<0$ and increases when $\bullet>0$.
\end{abstract}

Key words: Boundary-layer, Brownian motion, nanofluid, stretching sheet, thermophoresis, concentration

\section{INTRODUCTION}

The term nanofluid which was first coined by Choi (1995) in his bid to propose the new class of enhancing heat transfer fluid by dispersing nano-sized particles in a base fluid. Many pieces of research have been carried out on the notion behind the heat transfer enhancement by using nanofluids. The fluid flow over a stretching surface is indeed an essential and interesting case in engineering and sciences with an application such as metal spinning, wire drawing, extrusion and rubber sheets manufacturing amongst others. The convective heat transfer in nanofluids is an amazing area of interest by many researchers for its wide purview of application in both sciences and engineering, more can be found by Makinde and Aziz (2011). Mushtaq et al. (2014) investigated the radiative effects in the nanofluid flow due to solar energy using Runge-Kutta method. Their results show that both the temperature and wall temperature gradient increases with the increase of radiation parameter. Amongst other vital studies concerning the convective boundary layer flow are Aziz et al. (2012) and Makinde et al. (2013). Recently, Pal and Mandal (2015) investigated the hydromagnetic convective-radiative boundary layer flow of nanofluids caused by a non-linear vertical stretching/shrinking sheet with viscous dissipation numerically.

The term boundary layer flow is the fluid's flow region of a viscous fluid which is in the proximity of a body or a solid surface in contact with the fluid and in motion corresponding to the fluid. Very recently, Khazayinejad et al. (2016) studied the boundary-layer flow of a nanofluid past a porous moving semi-infinite plate using an optimal collocation method.

The Magnetohydrodynamic (MHD) nanofluid flows, being an electrically-conducting fluid have received a significant consideration due to its wide application in industrial technology, such as high temperature plasmas and purification of molten metals from non-metallic inclusions in metallurgy by the application of a magnetic field (Sing and Kumar, 2015; Mabood et al., 2015). He (1999) proposed a new perturbation method known as Homotopy Perturbation Method (HPM) which is a connection of the traditional perturbation method with homotopy as used in topology by Nayfeh (1993) and Krylovas (2008) presented asymptotic method for approximation of resonant interaction of nonlinear multidimensional hyperbolic waves using perturbation

Corresponding Author: R. Roslan, Faculty of Science, Technology and Human Development, Universiti Tun Hussein Onn Malaysia, 86400 Batu Pahat, Johor, Parit Raja, Malaysia 
method. This new method got a better advantage with the fact that it provides a uniformly suitable in a large region of asymptotic approximation of wave processes of order $0\left(\bullet^{-1}\right)$ where $\cdot$ is a small parameter.

The chemical reaction is quite a major platform required by many industrial applications in a manufacturing process. Naramgari and Sulochana (2016) studied the effects of both thermal radiation and chemical reaction on MHD flow over a permeable stretching/shrinking sheet of a nanofluid with suction/injection numerically using bvp $4 c$ with MATLAB package. Recently, many researchers such as Animasaun, (2015), Kasmani et al. (2016), Krishnamurthy et al. (2016) and Raju et al. (2016) had all made a fantastic effort in studying the effects of chemical reaction in relation to various cases in sciences.

In this study, our prime goal is to analyze the natural convective boundary layer flow, heat and mass transfer of nanofluid over a stretching sheet with convective boundary condition being the main mechanism. Some useful dimensionless quantities are used to transform the governing equations into ordinary differential equations. Both analytical and numerical techniques were used to derive the solution of the stated problem. The effect of chemical reaction along with all related parameters are investigated and presented in tabular and graphical forms, respectively.

\section{MATERIALS AND METHODS}

Problem formulation: Consider the two-dimensional $(\mathrm{x}, \mathrm{y})$ incompressible boundary-layer flow of a nanofluid past a heated stretching sheet situated at $\mathrm{y}=0$ with convective boundary condition. The two equal and opposite forces from the origin are the directions where the sheet is stretched along $\mathrm{x}$-axis and perpendicular to $\mathrm{y}$-axis. The velocity at the sheet surface (wall) is kept at the fixed origin along the distance $\mathrm{x}$, i.e., $\mathrm{U}_{\mathrm{w}}=\mathrm{ax}$ where $\mathrm{a}>0$ and $\mathrm{x}$ is the coordinate along the sheet, where the sheet velocity is zero as presented in Fig. 1. Let the fluid's free stream velocity be $\mathrm{U} .(\mathrm{x})=\mathrm{bx}$. The transverse magnetic field is subjected to the flow along $y>0$ normal to the fluid flow direction. Assume that the external electrical field is zero while the electrical field caused by the polarization of charges is negligible. Then the mass transfer analysis is performed subject to the chemical reaction as well as the other pertinent parameters effects. At the convective heating process, the sheet surface temperature $T_{w}$ goes with quiescent fluid temperature $\mathrm{T}_{\mathrm{f}}$. The ambient fluid's temperature is denoted by $\mathrm{T}$. The nanoparticle concentration is denoted by $\mathrm{C}$ while $\mathrm{C}_{\mathrm{w}}$ is the nanoparticle concentration at the wall and $\mathrm{C}$ is the ambient

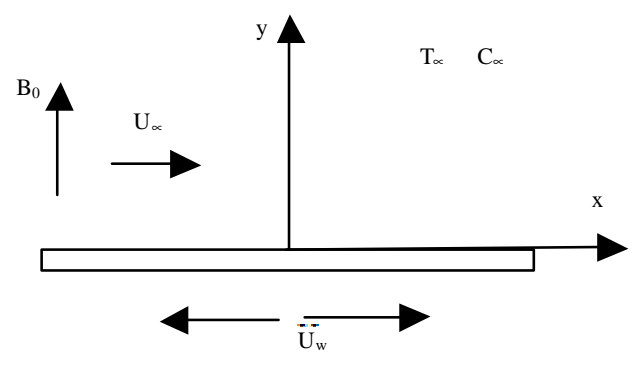

Fig. 1: Geometry of the problem

concentration. Based on the usual boundary layer assumptions, the equations controls the two-dimensional incompressible flow of a nanofluid in the presence of chemical reaction can be written as follows:

$$
\begin{gathered}
\frac{\partial \psi}{\partial \mathrm{y}} \frac{\partial^{2} \psi}{\partial \mathrm{x} \partial \mathrm{y} 2}-\frac{\partial \psi \partial^{2} \psi}{\partial \mathrm{x} \partial \mathrm{y}^{2}}=\mathrm{U}_{\infty} \frac{\partial \mathrm{u} \infty}{\partial \mathrm{x}}+\mathrm{vf} \frac{\partial^{3} \psi}{\partial \mathrm{y}^{3}}+\frac{\sigma_{\mathrm{g}} \mathrm{B}_{0}^{2}}{\rho_{\mathrm{f}}}\left(\mathrm{u}-\mathrm{U}_{\infty}\right) \\
\frac{\partial \psi}{\partial \mathrm{y}} \frac{\partial \mathrm{T}}{\partial \mathrm{x}}-\frac{\partial \psi}{\partial \mathrm{x}} \frac{\partial \mathrm{T}}{\partial \mathrm{y}}=\mathrm{a} \frac{\partial^{2} \mathrm{~T}}{\partial \mathrm{y}^{2}}+\frac{\mathrm{vf}}{\mathrm{cf}}\left(\frac{\partial^{2} \psi}{\partial \mathrm{y}^{2}}\right)^{2}+\frac{\sigma \mathrm{gB}}{(\mathrm{pc})_{\mathrm{f}}}(\mathrm{U} \infty-\mathrm{u})^{2}+ \\
\tau\left\{\mathrm{D}_{\mathrm{B}} \frac{\partial \mathrm{T}}{\partial \mathrm{y}} \frac{\partial \mathrm{c}}{\partial \mathrm{y}}+\frac{\mathrm{DT}}{\mathrm{T}}\left(\frac{\partial \mathrm{T}}{\partial \mathrm{y}}\right)^{2}\right\}+\frac{16 \sigma^{*} \mathrm{~T}^{3}}{\partial(\rho \mathrm{c})_{\mathrm{fk}}^{*}} \frac{\partial^{2} \mathrm{~T}}{\partial \mathrm{y}^{2}} \\
\frac{\partial \psi}{\partial \mathrm{y}} \frac{\partial \mathrm{c}}{\partial \mathrm{x}}-\frac{\partial \psi}{\partial \mathrm{x}} \frac{\partial \mathrm{c}}{\partial \mathrm{y}}=\mathrm{D}_{\mathrm{B}}-\frac{\partial^{2} \mathrm{c}}{\partial \mathrm{y}^{2}}+\frac{\mathrm{D}_{\mathrm{T}}}{\mathrm{T}_{\infty}} \frac{\partial^{2} \mathrm{~T}}{\partial \mathrm{y}^{2}}-\mathrm{k}_{1}(\mathrm{C}-\mathrm{C} \infty)
\end{gathered}
$$

Subject to the following boundary conditions:

$$
\begin{gathered}
\mathrm{u}=\mathrm{U}_{\mathrm{w}}(\mathrm{x})=\mathrm{ax}, \mathrm{v}=0,-\frac{\partial \mathrm{T}}{\partial \mathrm{y}}=\mathrm{h}\left(\mathrm{T}_{\mathrm{f}}-\mathrm{T}\right), \mathrm{C}=\mathrm{C}_{\mathrm{w}} \text {, at } \mathrm{y}=0, \\
\mathrm{u} \rightarrow \mathrm{U} \infty(\mathrm{x})-\mathrm{bx}, \mathrm{v}-0, \mathrm{~T}-\mathrm{T}_{\infty}, \mathrm{C}-\mathrm{C}_{\infty}, \text { as } \mathrm{y} \rightarrow \infty
\end{gathered}
$$

Here, the stream function $-(\mathrm{x}, \mathrm{y})$ is considered as $\mathrm{u}=\bullet \cdot / \mathrm{y}$ and $\mathrm{v}=\bullet / \bullet \mathrm{x}$ where $\mathrm{u}$ and $\mathrm{v}$ are the velocities in $\mathrm{x}$ and $\mathrm{y}$ directions, $\mathrm{U}$. is the free stream velocity, ${ }_{\mathrm{f}}$ is the kinematic viscosity of the fluid, $\bullet$ is the electrical conductivity, $\mathrm{B}_{0}$ is the magnetic field, $\bullet \mathrm{f}$ is the density of the nanofluid, $\mathrm{T}$ is the temperature $(\bullet \mathrm{C})_{\mathrm{f}}$ is the heat capacity of the base fluid, $\bullet$ is the density, $\bullet$ is the thermal diffusivity, $\mathrm{C}_{\mathrm{f}}$ is the skin friction coefficient of the fluid, $D_{B}$ is the Brownian diffusion, $D_{T}$ is the thermophoretic diffusion, $\mathrm{k}_{1}$ is the rate of chemical reaction, $\bullet=\left(\bullet \mathrm{C}_{\mathrm{p}} / \bullet \mathrm{C}\right) \mathrm{f}$ is the ratios of the effective heat capacity of the nanoparticle material to the heat capacity of the fluid, $\mathrm{q}_{\mathrm{r}}$ is the radiative heat flux, $\mathrm{k}$ is the thermal conductivity of base fluid, $h$ is the convective heat transfer coefficient, and $\mathrm{a}$ and $\mathrm{b}$ are constants. The Rosseland approximation 
(Rosseland, 1931) is used to evaluate the radiative heat flux in energy equation as $\mathrm{qr}=4 \cdot{ }^{*} / 3 \mathrm{k}^{*}, \cdot \mathrm{T}^{4} / \bullet \mathrm{y}$ where, $\bullet{ }^{*}$ and $\mathrm{k}^{*}$ are the Stefan-Boltzmann constant and the mean absorption number (Mahanthesh et al., 2016). The temperature variation amidst the flow is significantly limited and the expression $\mathrm{T} 4=4 \mathrm{~T}^{3} \cdot-3 \mathrm{~T}^{4}$. is considered in the energy equation as a linear function of temperature by using Taylor series expansion about $\mathrm{T}$. and by ignoring the higher-order terms. We now introduce the following dimensionless quantities (Khan and Pop, 2010):

$$
\begin{aligned}
& \eta=\sqrt{\mathrm{a} / \mathrm{vf}} y, \psi=\sqrt{\mathrm{av}_{\mathrm{f}}} \times f(\eta), u=\operatorname{axf}^{\prime}(\mathrm{n}), \\
& \mathrm{v}=\sqrt{\mathrm{av}_{\mathrm{f}}} \mathrm{f}(\eta), \theta(\eta)=\frac{\mathrm{T}-\mathrm{T}_{\infty}}{\mathrm{T}_{\mathrm{f}}-\mathrm{T}_{\mathrm{z}}}, \psi(\eta)-\frac{\mathrm{C}-\mathrm{C}_{\infty}}{\mathrm{C}_{\mathrm{w}}-\mathrm{C}_{\infty}}
\end{aligned}
$$

Using the similarity transformation quantities in Eq. 5, into Eq. 1-4, then we obtain the following transformed ordinary differential equations:

$$
\begin{gathered}
\mathrm{f}^{\mathrm{m}}+\mathrm{ff}^{\mathrm{n}}-\mathrm{f}^{\prime 2}+\mathrm{A}^{2}+\mathrm{M}\left(\mathrm{A}-\mathrm{f}^{\prime}\right)=0 \\
\left.\left[(1+\mathrm{N})\left(1+\left(\theta_{\mathrm{c}_{2}}-1\right) \theta\right)^{2}\right) \theta^{\prime}\right]^{\prime}+\operatorname{Pr}\left[\mathrm{f} \theta^{\prime}+\mathrm{Nb} \theta^{\prime} \varphi+\right. \\
\left.\mathrm{Nt} \theta^{\prime 2}+\mathrm{E}_{\mathrm{c}} \mathrm{f}^{\prime 2}+\mathrm{ME}_{\mathrm{c}}\left(\mathrm{A}-\mathrm{f}^{\prime}\right)^{2}\right]=0 \\
{\left[\left(1+\mathrm{N}\left(1+\left(\theta_{\mathrm{c}}-1\right) \theta\right)^{2}\right) \theta^{\prime}\right]^{\prime}+\operatorname{Pr}\left[\mathrm{f} \theta^{\prime}=0\right.} \\
\left.+\mathrm{Nb} \theta^{\prime} \varphi^{\prime}+\mathrm{Nt} \theta^{2}+\mathrm{Ecf} \mathrm{f}^{\prime 2}+\mathrm{MEc}\left(\mathrm{A}-\mathrm{f}^{\prime}\right)^{2}\right] \\
\varphi^{\prime \prime}+\mathrm{Lef} \varphi^{\prime}+\frac{\mathrm{Nt}}{\mathrm{Nb}} \theta^{\prime \prime}-\gamma \varphi=0
\end{gathered}
$$

where, prime represents differentiation with respect to the function $\bullet, M=\bullet . \mathrm{B}_{0}^{2} / \bullet{ }^{\bullet}$ is the magnetic parameter, $\mathrm{A}=\mathrm{b} / \mathrm{a}$ is the ratio of the rates of free stream velocity to the velocity of the stretching sheet, $\mathrm{N}=16{ }^{*} \mathrm{~T}^{3} \cdot / 3 \mathrm{kk}^{*}$ is the radiation parameter and $\operatorname{Pr}=\bullet \bullet$ is the Prandtl number. Here, the dimensionless temperature in Eq. 5 is written in the form $\mathrm{T}=\mathrm{T} .\left(1+\left(\bullet_{\mathrm{c}}-1\right) \bullet\right)$ where $\bullet_{\mathrm{c}}=\mathrm{T}_{\mathrm{f}} / \mathrm{T}$.. Considering the first and the last terms on the right hand side of Eq. 2, we therefore, write:

$$
\left.\left.\left.\left.\mathrm{a}\left(\frac{\partial}{\partial \mathrm{y}}\right)\left[\frac{\partial \mathrm{T}}{\partial \mathrm{y}}(1+\mathrm{N}) 1+\left(\theta_{\mathrm{c}}-1\right) \theta\right)^{3}\right)\right]=\frac{\mathrm{a}\left(\mathrm{T}_{\mathrm{f}}-\mathrm{T}_{\infty}\right)}{\operatorname{Pr}}\left[(1+\mathrm{N}) 1+\left(\theta_{\mathrm{c}}-1\right) \theta\right)^{3}\right) \theta^{\prime}\right]^{\prime}
$$

Equation 6-9 are subject to the following boundary conditions:

$$
\begin{gathered}
f(0)=0, f^{\prime}(0)=1, f^{\prime}(\infty) \rightarrow A, \theta^{\prime}(0)=-B i[1-\theta(0)](10) \\
\varphi(0)=1, \theta(\infty) \rightarrow 0, \varphi(\infty) \rightarrow 0
\end{gathered}
$$

The parameters involved in Eq. 6-11 are defined as:

$$
\begin{gathered}
L_{e} \frac{v_{f}}{D_{g}}, N b=\frac{\tau D_{g}\left(C_{w}-C_{\infty}\right)}{v_{f}}, N t=\frac{\tau D_{T}\left(T_{f}-T_{\infty}\right)}{v_{f} T_{\infty}}, B_{i}= \\
\frac{h\left(v_{f} / a\right)^{1 / 2}}{k}, E_{c}=\frac{U_{w}^{2}(x)}{C_{p}\left(T_{w}-T_{\infty}\right)},
\end{gathered}
$$

And:

$$
\gamma=\frac{\mathrm{k}_{1} \mathrm{u}_{\mathrm{f}}\left(\mathrm{C}_{\mathrm{w}}-\mathrm{C}_{\mathrm{w}}\right)}{\mathrm{D}_{\mathrm{B}} \mathrm{C}_{\mathrm{w}}{ }^{\mathrm{a}}}
$$

Where:

$\mathrm{L}_{\mathrm{e}}=$ The Lewis number

$N_{b}=$ The Brownian motion parameter

$N_{t}=$ The thermophoresis parameter

$\mathrm{B}_{\mathrm{i}}=$ The Biot number

$\mathrm{E}_{\mathrm{c}}=$ The Eckert number

- $\quad$ = The chemical reaction parameter

The quantities of practical interest are the Nusselt number, $\mathrm{Nu}$ and the Sherwood number, Sh. As we observed that $\mathrm{x}$-coordinate does not fit to be ignored from the temperature equation. Therefore, we strive for the possible local similarity solutions. With $\mathrm{q}_{\mathrm{w}}$ and $\mathrm{q}_{\mathrm{m}}$ being the wall heat flux and wall mass flux, respectively are given as follows:

$$
\begin{gathered}
\mathrm{q}_{\mathrm{w}}=-\mathrm{k}\left(\frac{\partial \mathrm{T}}{\partial \mathrm{y}}\right)_{\mathrm{y}=0}+(\mathrm{qr})_{\mathrm{w}}=-\mathrm{k}\left(\mathrm{T}_{\mathrm{w}}-\mathrm{T}_{\infty}\right)(\mathrm{a} / \mathrm{vf})^{\frac{1}{2}} \\
{\left[1+\mathrm{N} \theta_{\mathrm{c}}^{2}\right] \theta^{\prime}(0) \mathrm{q}_{\mathrm{m}}=-\mathrm{DB}\left(\frac{\partial \mathrm{c}}{\partial \mathrm{y}}\right)_{\mathrm{y}=0}=-\mathrm{DB}\left(\mathrm{C}_{\mathrm{w}}-\mathrm{C}_{\infty}\right)\left(\mathrm{a} / \mathrm{v}_{\mathrm{f}}\right)^{\frac{1}{2}} \varphi^{\prime}(0)}
\end{gathered}
$$

Using the expressions of both local Nusselt and Sherwood numbers as:

$$
\begin{gathered}
\mathrm{Nu}_{\mathrm{x}}=\frac{\mathrm{xq}_{\mathrm{w}}}{\mathrm{k}\left(\mathrm{T}_{\mathrm{w}}-\mathrm{T}_{\mathrm{c}}\right)} \Rightarrow-\sqrt{\operatorname{Rex}}\left[1-N \theta_{\mathrm{c}}^{2}\right] \theta^{\prime}(0)=\mathrm{Nur}, \\
\mathrm{Sh}=\frac{\mathrm{xq}_{\mathrm{m}}}{\mathrm{D}_{\mathrm{B}}\left(\mathrm{C}_{\mathrm{w}}-\mathrm{C}_{\infty}\right)} \Rightarrow \sqrt{\operatorname{Re}_{\mathrm{x}}} \varphi(0)=\mathrm{Shr}
\end{gathered}
$$

Homotopy perturbation solution: Here, we apply the HPM to nonlinear ordinary differential Eq. 6-9. Those equations are decomposed into linear $\mathrm{L}$ and non-linear $\mathrm{N}$ parts. We guess the linear part of the momentum equation to be $\mathrm{L}(\mathrm{u})=\mathrm{fl}$ "with $\mathrm{N}(\mathrm{u})=\mathrm{ff}^{2}+\mathrm{A}^{2}+\mathrm{M}(\mathrm{A}-\mathrm{f})$ and $g(r)=0$. While in the case of Eq. 14 because of the nonlinearity of the equation, we choose the linear operator $\mathrm{L}(\mathrm{u})=\bullet$ " with: 


$$
\begin{aligned}
\mathrm{N}(\mathrm{u})= & \mathrm{f} \theta^{\prime}+\mathrm{Nb} \theta^{\prime} \varphi^{\prime}+\frac{1}{\operatorname{Pr}}\left[\left(1+\mathrm{N}\left(1+\left(\theta_{\mathrm{c}}-1\right) \theta\right)^{2}\right) \theta^{\prime}\right]^{\prime}+ \\
& \mathrm{Nt}^{\prime 2}+\mathrm{Ecf}^{\prime 2}+\mathrm{MEc}\left(\mathrm{A}-\mathrm{f}^{\prime}\right)^{2}
\end{aligned}
$$

while from Eq. 9, we have $\mathrm{L}(\mathrm{u})=$ •" with $\mathrm{N}(\mathrm{u})=$ Lef ' $+(\mathrm{Nt} / \mathrm{Nb}) \bullet "-\cdots$. Following HPM, thus, we have:

$$
\begin{gathered}
\mathrm{H}(\mathrm{f}, \mathrm{p})=(1-\mathrm{p})\left[\mathrm{f}^{\prime \prime}\right]+\mathrm{p}\left[\mathrm{f}^{\prime \prime \prime}+\mathrm{f}^{\prime \prime}+\mathrm{ff} \mathrm{f}^{\prime \prime}-\mathrm{f}^{\prime 2}+\mathrm{A}^{2}+\mathrm{M}\left(\mathrm{A}-\mathrm{f}^{\prime}\right)\right] \\
\mathrm{H}(\theta, \mathrm{p})=(1-\mathrm{p}) \theta^{\prime \prime}+\mathrm{p}\left[\theta^{\prime \prime}+\left[\left(1+\mathrm{N}\left(1+\left(\theta_{\mathrm{c}}-1\right) \theta\right) 3\right) \theta^{\prime}\right]^{\prime}+\right. \\
\left.\operatorname{Prf} \theta^{\prime}+\operatorname{Pr} \operatorname{Nb} \theta^{\prime} \varphi^{\prime}+\operatorname{Pr} \mathrm{Nt} \theta^{\prime 2}+\operatorname{PrEcf}{ }^{\prime 2}+\operatorname{PrMEc}\left(\mathrm{A}-\mathrm{f}^{\prime}\right)^{2}\right]=0 \\
\mathrm{H}(\varphi, \mathrm{p})=(1-\mathrm{p}) \varphi^{\prime \prime}+\mathrm{p}\left[\varphi^{\prime \prime}+\operatorname{Lef} \varphi^{\prime}+\left(\frac{\mathrm{Nt}}{\mathrm{Nb}}\right) \theta^{\prime \prime}-\gamma \varphi\right]=0
\end{gathered}
$$

We consider:

$$
\begin{gathered}
f(\eta)=f^{0}+p f_{1}+p^{2} f_{2}+, \ldots, \\
\theta(\eta)=\theta_{0}+p \theta_{1}+p^{2} \theta_{2}+, \ldots \\
\varphi(\eta)=\varphi_{0}+p \varphi_{1}+p^{2} \varphi_{2}+, \ldots
\end{gathered}
$$

We, now substitute f, and $•$ from Eq. 16-18 into Eq. 14-16 and using the boundary conditions (Eq. 10), after some simplification and equating the like powers of $\mathrm{p}$ terms, we get the following nine set of equations:

$$
\begin{aligned}
& f_{0}^{\prime \prime \prime}=0, \theta_{0}^{\prime}=0, \varphi_{0}^{\prime \prime}=0, \\
& \mathrm{f}_{1}^{\prime \prime \prime}+\mathrm{f}_{0} \mathrm{f}_{0}^{\prime \prime}-\mathrm{f}_{0}^{\prime 2}+\mathrm{A}^{2}+\mathrm{M}\left(\mathrm{A}-\mathrm{f}_{0}^{\prime}\right)=0 \text {, } \\
& \mathrm{O}_{1}^{\prime \prime}+\frac{1}{\mathrm{pr}}\left[\left(1+\mathrm{N}\left(1+\left(\mathrm{O}_{\mathrm{c}}-1\right) 0_{0}\right)^{2}\right) 0_{0}^{\prime}\right]^{\prime}+\mathrm{f}_{0} \mathrm{O}_{0}^{\prime}+ \\
& \mathrm{Nb} \theta_{0}^{\prime} \varphi_{0}^{\prime}+\mathrm{Nt} \theta_{0}^{\prime 2}+\mathrm{Ecf}_{0}^{\prime 2}+\mathrm{MEc}\left(\mathrm{A}-\mathrm{f}_{0}^{\prime}\right)^{2}=0 \\
& \varphi_{1}^{\prime \prime}+\mathrm{L}_{\mathrm{e}} \mathrm{f}_{0} \varphi_{0}^{\prime}+(\mathrm{Nt} / \mathrm{Nb}) \theta_{0}^{\prime \prime}-\gamma \varphi_{0}=0 \\
& \mathrm{f}_{2}^{\prime \prime \prime}+\mathrm{f}_{1} \mathrm{f}_{0}^{\prime \prime}+\mathrm{fof}_{1}^{\prime \prime}-2 \mathrm{f}_{0}^{\prime} \mathrm{f}_{1}^{\prime}-\mathrm{Mf}_{1}^{\prime}=0+ \\
& \mathrm{f}_{1} \theta_{0}^{\prime}+\mathrm{f}_{0} \theta_{1}^{\prime}+\mathrm{Nb} \theta_{1}^{\prime} \varphi_{0}^{\prime}+2 \mathrm{Nt} \theta_{0}^{\prime} \theta_{1}^{\prime}+ \\
& 2 \mathrm{NtEcf}_{0}^{\prime \prime} \mathrm{f}_{1}^{\prime}-2 \mathrm{AEcMf} \mathrm{f}_{1}^{\prime}+2 \mathrm{MEcf}_{0} \mathrm{f}_{1}^{\prime}=0 \text {, } \\
& \theta_{2}^{\prime \prime}+\operatorname{Lef}_{1} \varphi_{0}^{\prime}+\operatorname{Lef}_{0} \varphi_{1}^{\prime}+(\mathrm{Nt} / \mathrm{Nb}) \theta_{1}^{\prime \prime}-\gamma \varphi_{1}=0
\end{aligned}
$$

which need to be solved subject to:

$$
\begin{aligned}
& \mathrm{f}_{0}(0)=0, \mathrm{f}_{0}^{\prime}(0)=1, \mathrm{f}_{0}^{\prime \prime}(0)=\alpha, \theta_{0}^{\prime}=\beta, \\
& \theta_{0}(0)=\left(\theta_{0}^{\prime}(0)+\mathrm{B}_{\mathrm{i}}\right) / \mathrm{B}_{\mathrm{i}}, \varphi_{0}(0)=1, \varphi_{0}^{\prime}(0)=\mathrm{q} \\
& \mathrm{f} 1(0)=0, \mathrm{f}_{1}^{\prime \prime}(0)=0, \mathrm{f}_{1}^{\prime \prime}(0)=0, \theta 1(0)=\theta_{1}^{\prime}(0) / \mathrm{B}_{\mathrm{i}}, \\
& \theta_{1}^{\prime}(0)=0, \varphi_{1}(0)=0, \varphi_{1}^{\prime}(0)=0 \\
& \mathrm{f}_{2}(0)=0, \mathrm{f}_{2}^{\prime}(0)=0, \mathrm{f}_{2}^{\prime \prime}(0) 0, \theta_{2}(0)=\theta_{2}^{\prime}(0) / \mathrm{B}_{\mathrm{i}} \\
& \theta_{2}^{\prime}(0)=0, \varphi_{2}(0)=0, \varphi_{2}^{\prime}(0)=0
\end{aligned}
$$

Solving a set of nine ODEs Eq. 19 with respect to their boundary conditions Eq. 20, we obtain:

$$
\begin{gathered}
f_{0}(\eta)=\eta+\frac{1}{2} \alpha \eta^{2} \\
\theta_{0}(\eta)=\left(1+\frac{\beta}{B_{i}}\right)+\beta \eta
\end{gathered}
$$

$f_{1}(\eta)=\eta^{3}\left(1+M-M A-A^{2}\right) / 6+a^{4}(1+M) / 24+\alpha^{2} \eta^{5} / 120$

$\theta_{1}(\eta)=\left\{\left(36 N \beta^{4}-108 N \theta_{c} \beta^{4}+108 N \theta_{c}^{2} \beta^{4}-36 N \theta_{c}^{3} \beta^{4}-\right.\right.$

$72 \mathrm{~N} \theta_{c} \beta^{2} \mathrm{~B}_{\mathrm{i}}+144 \mathrm{~N} \theta_{\mathrm{c}}^{2} \beta^{3} \mathrm{~B}_{\mathrm{i}}-72 \mathrm{~N} \theta_{\mathrm{c}}^{3} \beta^{3} \mathrm{~B}_{\mathrm{i}}-$

$12 \mathrm{EcM} \operatorname{PrB}_{\mathrm{i}}{ }^{2}-12 \mathrm{Ec} \operatorname{Pr} \alpha^{2} \mathrm{~B}_{\mathrm{i}}{ }^{2}-12 \mathrm{NbPrq} \beta \mathrm{B}_{\mathrm{i}}{ }^{2}-$

$12 \mathrm{Nt} \operatorname{Pr} \beta^{2} \mathrm{~B}_{\mathrm{i}}{ }^{2}+36 \mathrm{~N} \theta_{\mathrm{c}}^{2} \beta^{2} \mathrm{~B}_{\mathrm{i}}-36 \mathrm{~N} \theta_{\mathrm{c}}^{3} \beta^{2} \mathrm{~B}_{\mathrm{i}}{ }^{2}+$

$\left.24 \mathrm{EcM} \operatorname{PrB}_{\mathrm{i}}{ }^{2} \mathrm{~A}-12 \mathrm{EcM} \operatorname{PrB}_{\mathrm{i}}{ }^{2} \mathrm{~A}^{2}\right) \eta^{2}+$

$\left(24 N \beta^{4} \mathrm{~B}_{\mathrm{i}}-72 \mathrm{NO}_{\mathrm{c}} \beta^{4} \mathrm{~B}_{\mathrm{i}}+72 \mathrm{NO}_{\mathrm{c}}^{2} \beta^{4} \mathrm{~B}_{\mathrm{i}}-\right.$

$24 \mathrm{~N}_{\mathrm{c}}^{3} \beta^{4} \mathrm{~B}_{\mathrm{i}}-8 \mathrm{EcMPraB}{ }_{i}{ }^{2}-4 \operatorname{Pr}^{2} \mathrm{~B}_{\mathrm{i}}{ }^{2}-$

$24 \mathrm{~N} \theta c \beta^{3} \mathrm{~B}_{\mathrm{i}}{ }^{2}+48 \mathrm{~N} \theta_{\mathrm{c}}^{2} \beta^{3} \mathrm{~B}_{\mathrm{i}}-24 \mathrm{~N} \theta_{\mathrm{c}}^{3} \beta^{3} \mathrm{~B}_{\mathrm{i}}{ }^{2}+$

$\left.8 \mathrm{EcMPraB}_{\mathrm{i}}{ }^{2} \mathrm{~A}\right) \eta^{2}+\left(-2 \mathrm{EcM} \operatorname{Pr}^{2} \mathrm{~B}_{\mathrm{i}}{ }^{2}\right)-$

$\operatorname{Pr} \alpha \beta B_{i}{ }^{2}+6 N \beta^{4} B_{i}^{2}-18 N \theta c \beta^{4} B_{i}+$

$\left.\left.18 \mathrm{~N} \theta_{c}^{2} \beta^{4} \mathrm{~B}_{\mathrm{i}}-6 \mathrm{~N} \theta_{\mathrm{c}}^{3} \beta^{4} \mathrm{~B}_{\mathrm{i}}\right) \eta^{4}\right\} / 24 \mathrm{~B}_{\mathrm{i}}{ }^{2}$

$$
\begin{gathered}
\varphi_{1}(\eta)=\frac{1}{2} \beta \gamma \eta^{2}-\frac{1}{6} \text { Leq }^{3}-\frac{1}{24}{\text { Leq } \alpha \eta^{4}}^{4} \\
\mathrm{f}_{2}(\eta)=\alpha \eta^{3}\left(-1-2 \mathrm{M}-\mathrm{M}^{2}-\mathrm{AM}-\mathrm{A}^{2}\right) / \\
6-\alpha^{2} \eta^{4}(1+\mathrm{M}) / 12-\alpha^{3} \eta^{5} / 60
\end{gathered}
$$

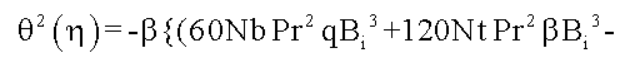
$\left.60 \operatorname{PrB}_{\mathrm{i}}{ }^{3}+\ldots\right) \eta^{2}+\left(20 \operatorname{Pr}^{2} \mathrm{~B}_{\mathrm{i}}{ }^{3}+20 \mathrm{Nb}^{2} \operatorname{Pr}^{2} \mathrm{q} \mathrm{B}_{\mathrm{i}}{ }^{3}+\right.$ $\left.40 \mathrm{Nt} \operatorname{Pr}^{2} \alpha \beta \mathrm{B}_{\mathrm{i}}{ }^{3}+\ldots\right) \eta^{3}+\left(15 \operatorname{Pr}^{2} \alpha \mathrm{B}_{\mathrm{i}}{ }^{3}+15(2-\right.$ $\left.\left.5 \theta_{\mathrm{c}}+3 \theta_{\mathrm{c}}^{2}\right) \beta^{2} \mathrm{~B}_{\mathrm{i}}{ }^{3}+\ldots\right) \eta^{4}+2 \mathrm{EcM} \operatorname{Pr} / \beta\left(3 \operatorname{Pr} \alpha^{3} \mathrm{~B}_{\mathrm{i}}{ }^{3}+\right.$ $\left.\left.108 \mathrm{~N}^{3} \theta_{\mathrm{c}}^{3} \beta^{5} \alpha^{1} \mathrm{~B}_{\mathrm{i}}{ }^{3}+\ldots\right) \eta^{5}\right\} / 120 \mathrm{~B}_{\mathrm{i}}{ }^{3}$

$$
\begin{aligned}
& \varphi_{2}(\eta)=\eta^{2}\left(\text { Leq-LeMq- } \frac{2}{\mathrm{Nb}} \text { EcMNt Pr } \alpha^{2}-\right. \\
& \frac{1}{\mathrm{Nb}} \mathrm{Nt} \operatorname{Pr} \alpha \beta+\frac{6}{\mathrm{Nb}} \mathrm{NtN}^{4}-\frac{18}{\mathrm{Nb}} \mathrm{NtN}_{\mathrm{c}} \beta^{4}+ \\
& \frac{10}{\mathrm{Nb}} \mathrm{NtN} \theta_{\mathrm{c}}^{z} \beta^{4}-\frac{6}{\mathrm{Nb}} \mathrm{NtN} \theta_{\mathrm{c}}^{3} \beta^{4}+\mathrm{Leq} \gamma- \\
& \text { LeMqA-LeqA } \left.{ }^{2}\right) / 2+\eta^{3}\left(- \text { Le }^{2} \mathrm{q}-\right. \\
& \text { Leq } \alpha+\text { LeMq } \alpha+\text { Leq } \alpha \text { )/6- } \\
& \frac{\eta^{5}}{40} \mathrm{Le}^{2} \mathrm{q} \alpha^{2}+\eta^{4}\left(-3 \mathrm{Le}^{2} \mathrm{q} \alpha+\right) / 24
\end{aligned}
$$


Table 1: Comparison of values of reduced Nusselt number, (Nur)- $\cdot(0)$ and reduced Sherwood number, (Shr)- '(0) with Mushtaq et al. (2014) when and $\mathrm{Nt}=\mathrm{Nb}=0.05$ and $\mathrm{N}=\boldsymbol{\bullet}=0$

\begin{tabular}{|c|c|c|c|c|c|c|}
\hline \multirow[b]{2}{*}{$\underline{P_{x}}$} & \multirow[b]{2}{*}{$\mathrm{B}_{\mathrm{i}}$} & \multirow[b]{2}{*}{$\mathrm{L}_{\mathrm{e}}$} & \multicolumn{2}{|c|}{ Mushtaq et al. (2014) } & \multicolumn{2}{|c|}{ Present HPM-Pade } \\
\hline & & & $\bullet(0)$ & $-\bullet '(0)$ & $\stackrel{\bullet}{-}(0)$ & $-\bullet^{\prime}(0)$ \\
\hline 1 & 0.1 & 5 & 0.0789 & 1.5476 & 0.07892 & 1.54750 \\
\hline 2 & 0.1 & 5 & 0.0806 & 1.5554 & 0.08068 & 1.55554 \\
\hline 5 & 0.1 & 5 & 0.0734 & 1.5983 & 0.07339 & 1.59790 \\
\hline 10 & 0.1 & 5 & 0.0386 & 1.7293 & 0.03857 & 1.72927 \\
\hline 5 & 1 & 5 & 0.1475 & 1.6913 & 0.14754 & 1.69136 \\
\hline 5 & 10 & 5 & 0.1549 & 1.7122 & 0.1 & 1.71223 \\
\hline 5 & 100 & 5 & 0.1555 & 1.7143 & 0.15543 & 1.71441 \\
\hline 5 & - & 5 & 0.1557 & 1.7146 & - & - \\
\hline 5 & 0.1 & 10 & 0.0646 & 2.3920 & 0.06466 & 2.39211 \\
\hline$\underline{5}$ & 0.1 & 15 & 0.0570 & 2.9900 & 0.05710 & 2.98988 \\
\hline
\end{tabular}

Table 2: Comparison between the computed values of Runge-Kutta fourth order and HPM-Pade for $\bullet \cdot(0)$ and $\bullet \cdot(0)$ when $P_{r}=7, M=A=$ $\mathrm{N}=0.3, \mathrm{Ec}=0.1, \mathrm{Le}=5 \bullet_{\mathrm{c}}=1.5, \mathrm{Nb}=0.1$ and $\mathrm{Bt}=0.1$

\begin{tabular}{|c|c|c|c|c|c|}
\hline \multirow[b]{2}{*}{$\mathrm{Nt}$} & \multirow[b]{2}{*}{ - } & \multicolumn{2}{|c|}{ Runge-Kutta } & \multicolumn{2}{|c|}{ HPM-Pade [3/3] } \\
\hline & & $\bullet^{\prime}(0)$ & $\bullet^{\prime}(0)$ & • ' $(0)$ & .' \\
\hline \multirow[t]{2}{*}{$\overline{0.1}$} & -1.0 & 0.08168 & 1.28137 & - & 1.28140 \\
\hline & 1.0 & 0.08119 & 1.91374 & 0.08119 & 1.91392 \\
\hline \multirow[t]{2}{*}{0.2} & -1.0 & 0.08115 & 1.28524 & - & 1.28533 \\
\hline & 1.0 & 0.08054 & 1.96240 & 0.08054 & 1.96251 \\
\hline \multirow[t]{2}{*}{0.3} & -1.0 & 0.08060 & 1.29370 & - & 1.29384 \\
\hline & 1.0 & 0.07980 & 2.01887 & 0.07981 & 2.01891 \\
\hline \multirow[t]{2}{*}{0.4} & -1.0 & 0.07992 & 1.30644 & - & 1.30590 \\
\hline & 1.0 & 0.07897 & 2.08438 & 0.07896 & 2.08441 \\
\hline \multirow[t]{2}{*}{0.5} & -1.0 & 0.07920 & 1.32497 & - & 1.32518 \\
\hline & 1.0 & 0.07803 & 2.16053 & 0.07811 & 2.16090 \\
\hline
\end{tabular}

We ignored to write the other part of the approximations in Eq. 28 due to the fact that they looked a bit vast. Finally, the required solutions of Eq. 6-9, subject to the boundary conditions (Eq. 10) can be obtained using Eq. 21-29 into expressions Eq. 16-18.

Pade approximation: A Pade approximation is the rational function of a given order of two polynomials generated from the coefficients of the Taylor series expansion of a function. It is glaringly obvious, that this notion gives an advantage of handling a polynomial approximation into rational polynomials. Thus, the integration of homotopy perturbation method with the Padé approximation gives an efficient tool for managing ordinary differential equation of boundary value problems on semi-infinite or infinite domains (Boyd, 1997; Bararnia et al., 2012). Furthermore, the Pade approximation mostly converges on the entire real axis if $\mathrm{f}(\bullet), \bullet(\bullet)$ and $\bullet(\bullet)$ are free of singularities on the real axis. Therefore, we construct the diagonal approximants, since, it certainly gives the most accurate approximants. Even though, the Pade approximants can be easily found in built-in functions in most of the manipulation languages like Maple and Mathematica, respectively. Hence, the missing boundary conditions - ' $(0)=\bullet$ and $\bullet '(0)=\mathrm{q}$ from our general problem are found by setting the coefficients of the highest power of the

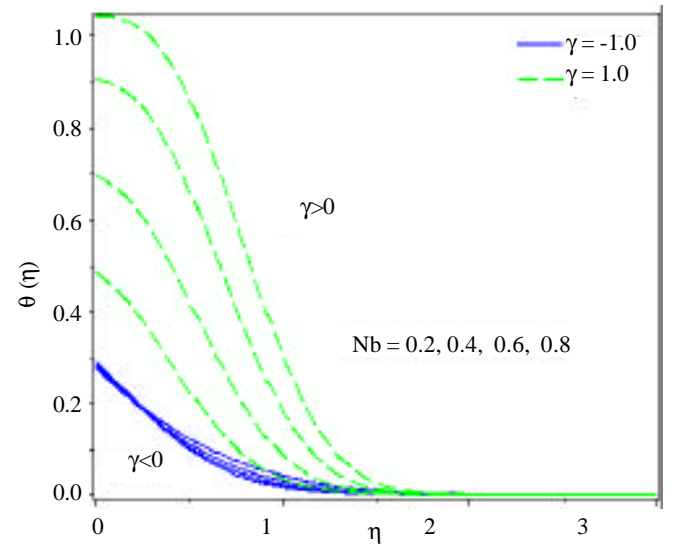

Fig. 2: Effect of $\mathrm{Nb}$ on $\bullet$ when $\operatorname{Pr}=7, \mathrm{~N}=\mathrm{A}=\mathrm{M}=\mathrm{B}_{\mathrm{i}}=0.5$ and $\bullet_{\mathrm{c}}=1.5$

independent variable $\cdot$ equal to zero after we must have used the boundary conditions $\mathrm{f}^{\prime}(\bullet)=\mathrm{A}, \cdot(\bullet)=0$ and $\bullet(\bullet)=0$ as depicted in Table 1 and 2 .

\section{RESULTS AND DISCUSSION}

We solved the nonlinear ordinary differential (Eq. 6-9) with respect to their boundary conditions Eq. 10, using HPM-Pade and validated by fourth order Runge-Kutta method with shooting method in Maple 18. The accuracy of our results is displayed in Table 1 at the various values of parameters involved while considering the chemical reaction parameter $\bullet=0$. Thus, a very fascinate agreement between the present and previous studies were attained. The results displayed in Table 2 show that HPM-Pade is matching the numerical approach. It can be observed in Table 2 that, we have presented the impact caused by both Brownian motion and thermophoresis on the dimensionless heat transfer rate - $\bullet '(0)$ when $\mathrm{Nb}$ at a certain value and varying the values of $\mathrm{Nt}$ along with both types of chemical reaction (i.e., generative and destructive). This resulted to the decrease in the reduced Nusselt number Nur significantly. The dimensionless mass transfer rate - ' $(0)$ notably increases while the $\mathrm{Nb}$ being kept at certain value and $\mathrm{Nt}$ intensifies from (0.1-0.5) along with both generative and destructive chemical reaction parameter values, respectively. Figure 2 shows an effect of $\mathrm{Nb}$ to the temperature profile been at various values in the presence of both types chemical reaction. The Brownian motion in nanofluid occurs by the virtue of the nano-sized of the particles which plays an essential role in the heat transfer. So, in this regard, the temperature increases with an increase of $\mathrm{Nb}$ when $\bullet>0$ and the temperature decreases with an increase of $\mathrm{Nb}$ when $\bullet<0$, respectively. It is well observed 
J. Eng. Applied Sci., 14 (Special Issue 6): 9224-9231, 2019

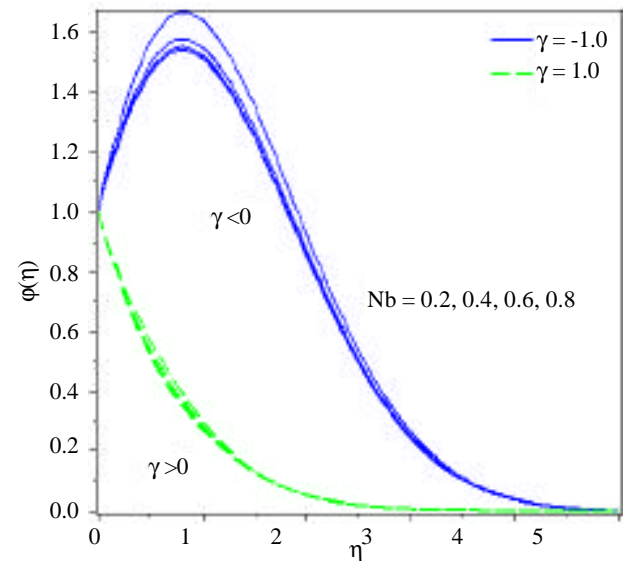

Fig. 3: Effects of $\mathrm{Nb}$ on when $\operatorname{Pr}=7 \mathrm{~N}=\mathrm{A}=\mathrm{MB}_{\mathrm{i}}=0.5$, $\mathrm{Le}=1$ and $\cdot{ }_{\mathrm{c}}=1.5$

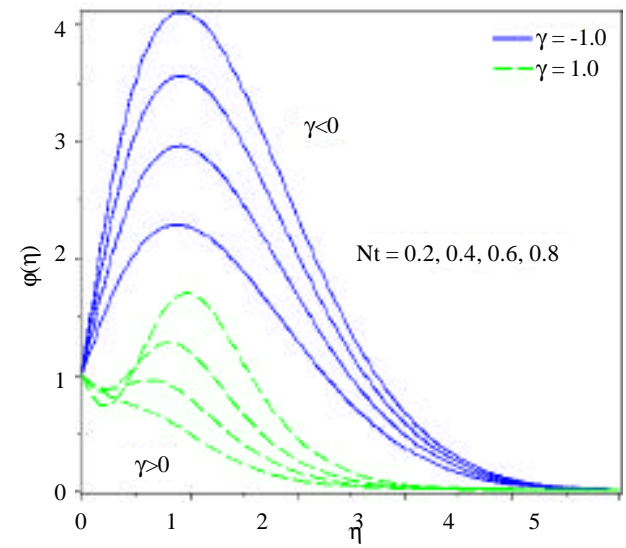

Fig. 4: Effects of $\mathrm{Nt}$ on when $\operatorname{Pr}=7, \mathrm{~N}=\mathrm{A}=\mathrm{M}=\mathrm{B}_{\mathrm{i}}=$ $0.5,{ }_{\mathrm{c}}=1.5$

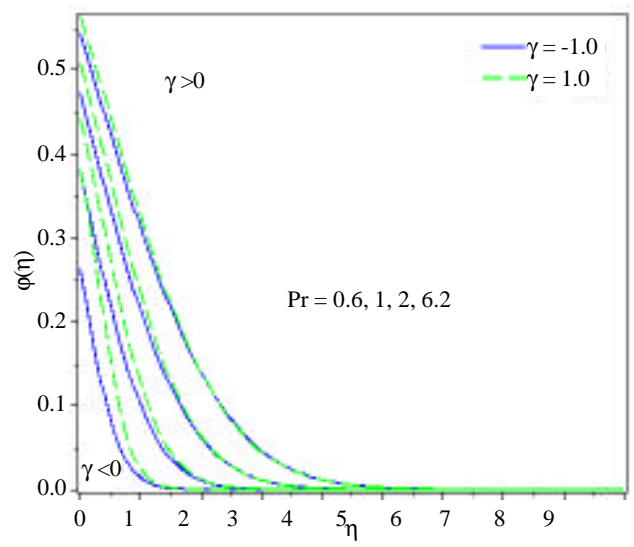

Fig. 5: Effects of $\operatorname{Pr}$ on when $\mathrm{N}=\mathrm{A}=\mathrm{M}=\mathrm{B}_{\mathrm{i}}=0.5, \mathrm{~L}_{\mathrm{e}}=$ 1 and $\bullet_{c}=1.5$

that in Fig. 3, the increase in the values of $\mathrm{Nb}$ leads to the drastic decrease in the nanoparticles volume fraction profile $\bullet(\bullet)$ with respect to the generative chemical

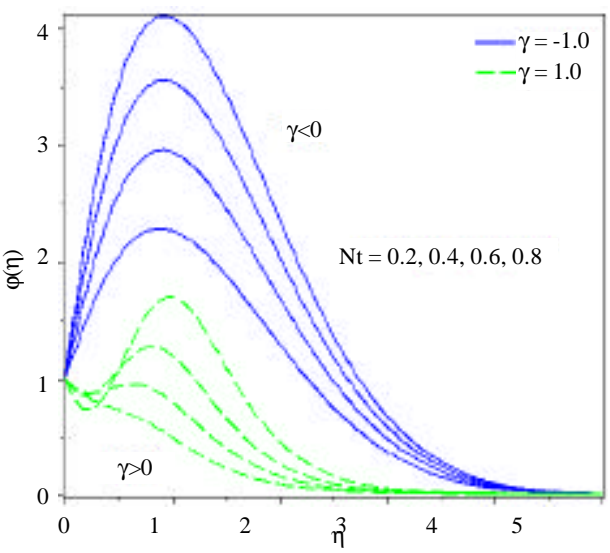

Fig. 6: Effects of $B_{i}$ on when $\operatorname{Pr}=7, N=A=M=0.5, N b$

$$
=\mathrm{Nt}=0.1, \mathrm{E}_{\mathrm{c}}=0.2, \mathrm{~L}_{\mathrm{e}}=1 \text { and } \bullet_{\mathrm{c}}=1.5
$$

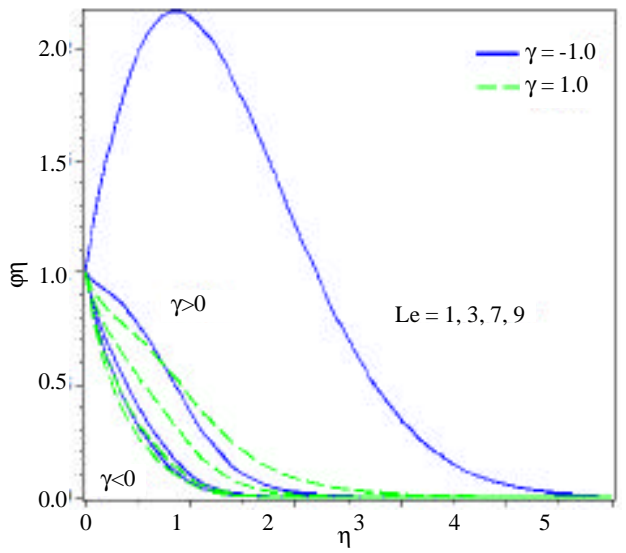

Fig. 7: Effects of Le on when $\operatorname{Pr}=7, \mathrm{~N}=\mathrm{A}=\mathrm{M}=\mathrm{B}_{\mathrm{i}}=$ 0.5 and ${ }_{c}=1.5$

reaction $-<0$ but it increases when $\cdot>0$ along the stretching sheet. Besides, the concentration boundary layer has been significantly affected as the values of $\mathrm{Nt}$ is increasing, this results to the decrease in nanoparticles concentration profile $\bullet(\bullet)$ when $\bullet<0$ and reduces when $->0$ as depicted in Fig. 4. The Prandtl number influence on the boundary layer temperature is presented in Fig. 5 where it worth mention that, as the fluid considered is indeed at high viscosity, thus, the Prandtl number with both small and large values were chosen, so as to see the nature of their impact upon the thermal boundary layer. It is found that as $\mathrm{Pr}$ increases, the temperature of the fluid within the boundary layer increases and decreases rapidly when $\bullet<0$ and $\bullet>0$, respectively. Figure 6 shows that when $\mathrm{B}_{\mathrm{i}}$ increases and $\bullet<0$ resulted to the increase in the nanoparticles concentration profiles significantly. While it keeps reducing towards the wall when $\mathrm{Bi}$ is increasing and $\bullet>0$. The nanoparticles 


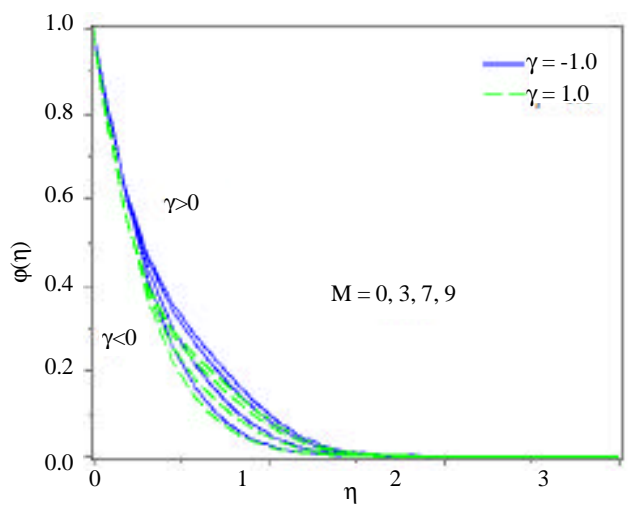

Fig. 8: Effects of $\mathrm{M}$ on when $\operatorname{Pr}=7, \mathrm{~N}-\mathrm{A}-\mathrm{A}-\mathrm{B}_{\mathrm{i}}-0.5, \mathrm{Nb}=$ $\mathrm{Nt}=0.1, \mathrm{~L}_{\mathrm{e}}=10, \mathrm{Ec}=0.2$ and ${ }_{\mathrm{c}}=1.5$

concentration decreases with an increase of Le when $\bullet>0$ and reverse is the case when $\bullet>0$ as the nanoparticles concentration rises as presented in Fig. 7. The nanoparticles concentration decreases with an increase in the values of $\mathrm{M}$ when $\bullet<0$ but increases when $\bullet>0$ as depicted in Fig. 8.

\section{CONCLUSION}

In this study, the influence of chemical reaction parameter have been studied, along with other pertinent parameters such as Brownian motion parameter $\mathrm{Nb}$ thermophoresis parameter Nt, $\mathrm{Pr}$ and $t 1$ number $\mathrm{Pr}$, Eckert number $E_{o}$ Biot number $B_{i j}$ Lewis number $L_{e}$ and magnetic parameter $\mathrm{M}$ on the flow field and natural convective boundary layer flow of a nanofluid past a stretching sheet in the presence of chemical reaction. The analysis to this problem was made both analytically and numerically. Our results found to be in an excellent agreement with previous study by Mushtaq et al. (2014) while some distinct parameters in this study being limited to zero. The results found in this study can be summarized as follows: The temperature and thermal boundary layer thickness increase with an increase in the values of Brownian motion $\mathrm{Nb}$ when $\bullet>0$ but the nanoparticles concentration decreases when $\bullet<0$; The destructive chemical reaction - $<0$ with an increase in the thermophoresis parameter $\mathrm{Nt}$, fascinatingly leads to the decrease in nanoparticle concentration profile; Increasing the values of Prandtl number $\mathrm{Pr}$ results to the significance increase in the temperature profiles when $\cdot>0$; The presence of the parameter $\bullet>0$, results to the significance decrease in the nanoparticles concentration; An increase in the Lewis number Le reduces the nanoparticles concentration profiles rapidly when $\bullet<0$; The nanoparticles concentration profiles fantastically keep decreasing in the case of $\bullet<0$ with an increase in the magnetic parameter $\mathrm{M}$;
It is also found that the reduced Nusselt number decreases at the various values of parameters involved while the reduced Sherwood number increases significantly.

\section{ACKNOWLEDGEMENTS}

The researchers would like to express their profound gratitude to the Universiti Tun Hussein Onn Malaysia for the financial aid received from the Grant GIPS/ U191, FRGS/1434 and RSGS/U112.

\section{REFERENCES}

Animasaun, I.L., 2015. Effects of thermophoresis, variable viscosity and thermal conductivity on free convective heat and mass transfer of non-darcian $\mathrm{MHD}$ dissipative Casson fluid flow with suction and nth order of chemical reaction. J. Nige. Math. Soc., 34: 11-31.

Aziz, A., W.A. Khan and I. Pop, 2012. Free convection boundary layer flow past a horizontal flat plate embedded in porous medium filled by nanofluid containing gyrotactic microorganisms. Int. J. Therm. Sci., 56: 48-57.

Bararnia, H., E. Ghasemi, S. Soleimani, A.R. Ghotbi and D.D. Ganji, 2012. Solution of the Falkner-Skan wedge flow by HPM-Pade' method. Adv. Eng. Software, 43: 44-52.

Boyd, J.P., 1997. Pade approximant algorithm for solving nonlinear ordinary differential equation boundary value problems on an unbounded domain. Comput. Phys., 11: 299-303.

Choi, S.U.S., 1995. Enhancing Thermal Conductivity of Fluids with Nanoparticles. In: Developments and Applications of Non-Newtonian Flows, Siginer, D.A. and H.P. Wang (Eds.). American Society of Mechanical Engineers, New York, pp: 99-105.

He, J.H., 1999. Homotopy perturbation technique. Comput. Methods Applied Mech. Eng., 178: 257-262.

Kasmani, R.M., S. Sivasankaran, M. Bhuvaneswari and Z. Siri, 2016. Effect of chemical reaction on convective heat transfer of boundary layer flow in nanofluid over a wedge with heat generation/absorption and suction. J. Appl. Fluid Mech., 9: 379-388.

Khan, W.A. and I. Pop, 2010. Boundary-layer flow of a nanofluid past a stretching sheet. Int. J. Heat Mass Transfer, 53: 2477-2483.

Khazayinejad, M., M. Hatami, D. Jing, M. Khaki and G. Domairry, 2016. Boundary layer flow analysis of a nanofluid past a porous moving semi-infinite flat plate by optimal collocation method. Powder Technol., 301: 34-43. 
Krishnamurthy, M.R., B.C. Prasannakumara, B.J. Gireesha and R.S.R. Gorla, 2016. Effect of chemical reaction on MHD boundary layer flow and melting heat transfer of Williamson nanofluid in porous medium. Eng. Sci. Technol. Int. J., 19: 53-61.

Krylovas, A., 2008. Asymptotic method for approximation of resonant interaction of nonlinear multidimensional hyperbolic waves. Math. Modell. Anal., 13: 47-54.

Mabood, F., W.A. Khan and A.M. Ismail, 2015. MHD boundary layer flow and heat transfer of nanofluids over a nonlinear stretching sheet: A numerical study. J. Magn. Magn. Mater., 374: 569-576.

Mahanthesh, B., B.J. Gireesha and R.S.R. Gorla, 2016. Nonlinear radiative heat transfer in MHD three-dimensional flow of water based nanofluid over a non-linearly stretching sheet with convective boundary condition. J. Niger. Math. Soc., 35: 178-198.

Makinde, O.D. and A. Aziz, 2011. Boundary layer flow of a nanofluid past a stretching sheet with a convective boundary condition. Int. J. Therm. Sci., 50: 1326-1332.

Makinde, O.D., W.A. Khan and Z.H. Khan, 2013. Buoyancy effects on MHD stagnation point flow and heat transfer of a nanofluid past a convectively heated stretching/shrinking sheet. Intl. J. Heat Mass Transfer, 62: 526-533.
Mushtaq, A., M. Mustafa, T. Hayat and A. Alsaedi, 2014. Nonlinear radiative heat transfer in the flow of nanofluid due to solar energy: A numerical study. J. Taiwan Inst. Chem. Eng., 45: 1176-1183.

Naramgari, S. and C. Sulochana, 2016. MHD flow over a permeable stretching/shrinking sheet of a nanofluid with suction/injection. Alexandria Eng. J., 55: 819-827.

Nayfeh, A.H., 1993. Introduction to Perturbation Techniques. Wiley-Interscience, New York.

Pal, D. and G. Mandal, 2015. Hydromagnetic convective: Radiative boundary layer flow of nanofluids induced by a non-linear vertical stretching/shrinking sheet with viscous-Ohmic dissipation. Powder Technol., 279: 61-74.

Raju, C.S.K., N. Sandeep, V. Sugunamma, M.J. Babu and J.R. Reddy, 2016. Heat and mass transfer in magnetohydrodynamic Casson fluid over an exponentially permeable stretching surface. Eng. Sci. Technol. Int. J., 19: 45-52.

Singh, P. and M. Kumar, 2015. Mass transfer in MHD flow of alumina water nanofluid over a flat plate under slip conditions. Alexandria Eng. J., 54: 383-387. 